Blome, Tanja; Dold, Christian; El Zohbi, Juliane; Köhnke, Fiona; Preuschmann, Swantje; Steuri, Bettina; Sun, Jianing; Rechid, Diana; Schultz, Martin; Jacob, Daniela

\title{
Neue digitale Formate für die Kommunikation von CO2-Einsparungspotentialen für Deutschland
}

Zur Verfügung gestellt in Kooperation mit / Provided in Cooperation with:

Deutsche Meteorologische Gesellschaft

Verfügbar unter / Available at:

https://hdl.handle.net/20.500.11970/107748

Vorgeschlagene Zitierweise / Suggested citation:

Blome, Tanja; Dold, Christian; El Zohbi, Juliane; Köhnke, Fiona; Preuschmann, Swantje; Steuri, Bettina; Sun, Jianing; Rechid, Diana; Schultz, Martin; Jacob, Daniela (2021): Neue digitale Formate für die Kommunikation von CO2-Einsparungspotentialen für Deutschland. Poster präsentiert bei: 12. Deutsche Klimatagung, Online-Tagung, 15. bis 18. März 2021. https://doi.org/10.5194/dkt-12-6.

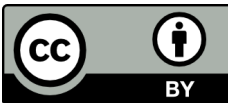




\section{HELMHOLTZ}

\section{: CLIMATE INITIATIVE}

\section{Neue digitale Formate für die Kommunikation von C02-Einsparungspotentialen für Deutschland}

Tanja Blome' ${ }^{1}$, Christian Dold², Juliane El Zohbi ${ }^{1}$, Fiona Köhnke ${ }^{1}$, Swantje Preuschmann ${ }^{1}$, Bettina Steuri ${ }^{1}$, Jianing Sun ${ }^{3}$, Diana Rechid $^{1}$, Martin Schultz ${ }^{3}$, Daniela Jacob ${ }^{1}$, and Netto-Null Team (Cluster I Helmholtz-Klima-Initiative)

1) Climate Service Center Germany (GERICS) / HZG, Geesthacht, Germany

2) Forschungszentrum Jülich, IBG3, Jülich, Germany

3) Forschungszentrum Jülich, Jülich Supercomputing Centt) Jülich, Germany 


\section{Helmholtz-Klima-Initiative}

\section{Fakten zum Projekt}

WAS Förderung bereichsübergreifender Forschung in den Schwerpunkten Reduzierung von Emissionen \& Anpassung an Klimafolgen in der Helmholtz-Gemeinschaft. Beschreiten neuer Wege in Bezug auf wissenschaftliche Methoden und Kommunikation.

WIE 3 Cluster, 13 Forschungsprojekte

Finanzielle Förderung durch den Impuls- und Vernetzungsfond der Helmholtz-Gemeinschaft

WER $\quad 15$ Helmholtz-Forschungszentren 6 Forschungsbereiche

Ausgewählte externe Partner:innen \& Stakeholder

WANN Juli 2019 - Dezember 2021

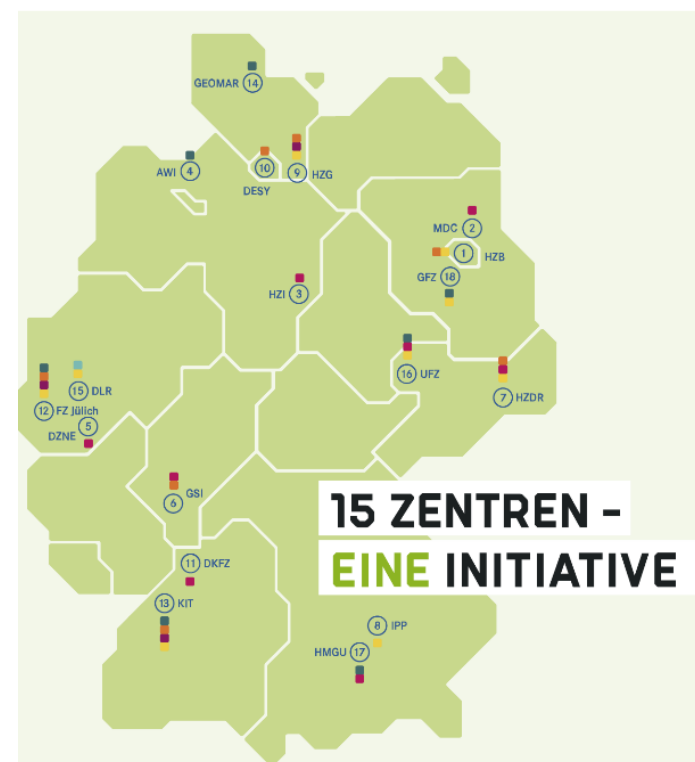

https://www.helmholtz-klima.de 


\section{Helmholtz-Klima-Initiative}

\section{Projektstruktur: Drei zusammenarbeitende Cluster}

Cluster I: Netto-Null-2050

Koordination: Climate Service Center Germany (GERICS) I Helmholtz-Zentrum Geesthacht

Die Entwicklung von einem wissenschaftsbasierten Handlungsrahmen zur Unterstützung einer Minderungsstrategie, die zu einem CO2-neutralen Deutschland im Jahr 2050 führt.

https://www.netto-null.org/

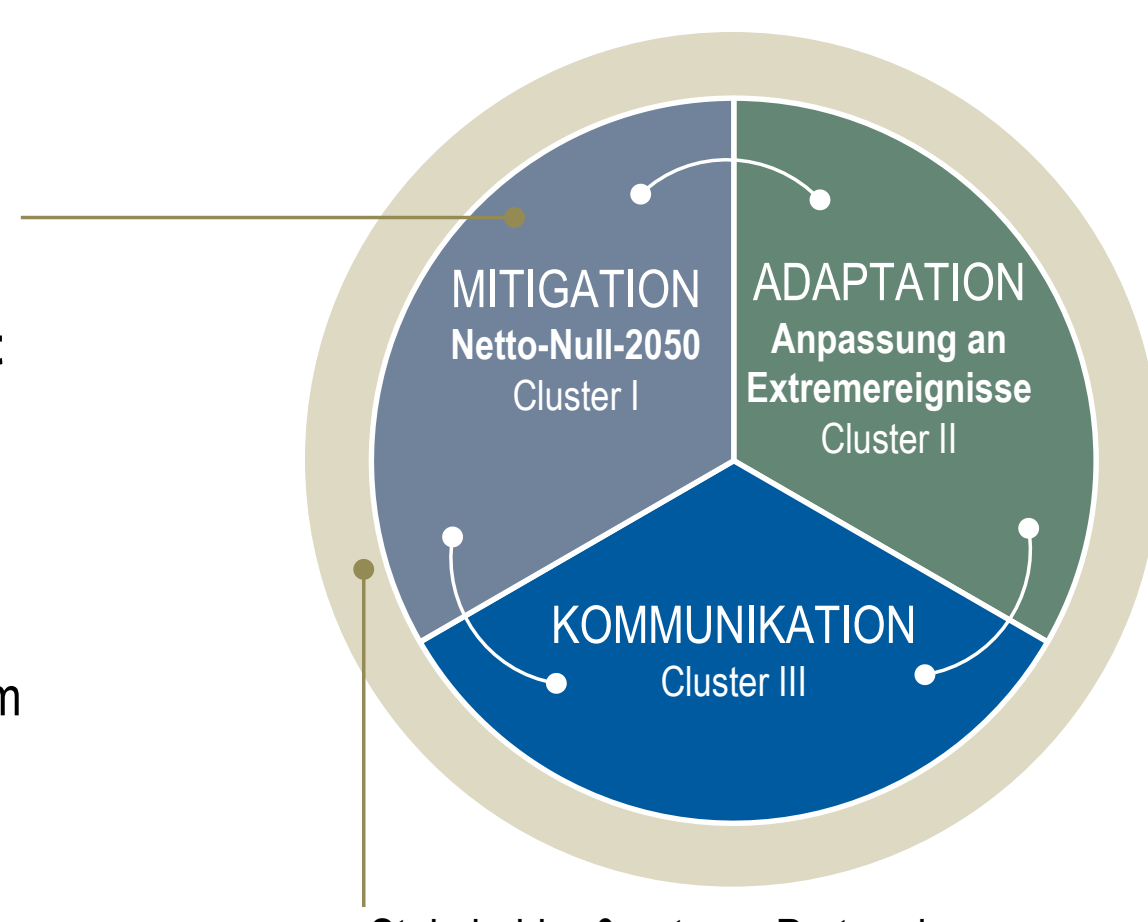

Stakeholder \& externe Partner:innen 


\section{Komplexe Ergebnisse der Forschung verständlich zeigen}

Bodenkohlenstoff-App und Netto-Null Web-Atlas

\section{Ziel}

- Beide Formate haben das Ziel die Nutzer:innen in Entscheidungen und bei der Entwicklung von Strategien zu einem CO2-neutralen Deutschland bis 2050 zu unterstützen.

\section{Bodenkohlenstoff-App}

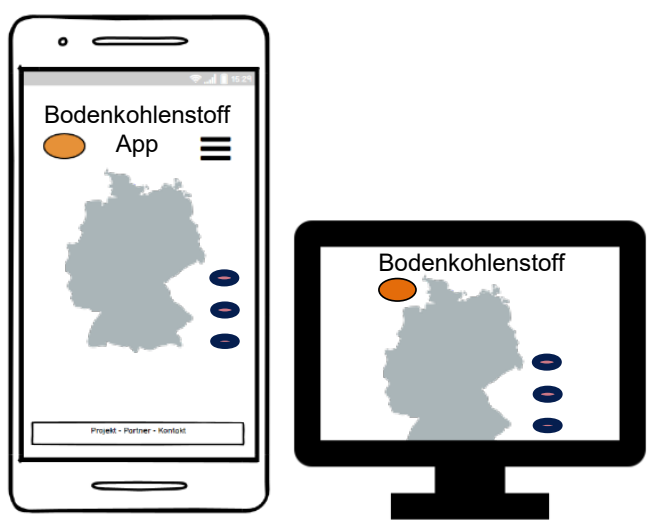

\section{Netto-Null-2050 Web-Atlas}

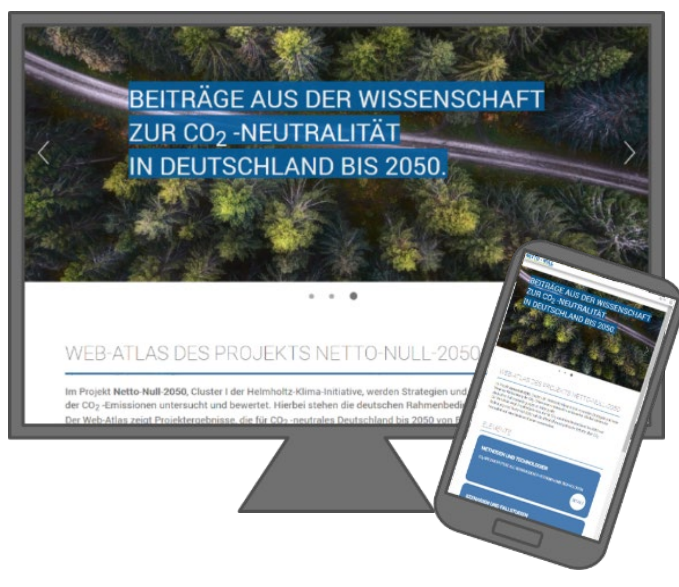




\section{Bodenkohlenstoff-App}

\section{Ziel und Nutzer:innengruppen}

\section{Spezifisches Ziel}

- Bereitstellung lokaler Informationen zum Potenzial der Bodenkohlenstoffspeicherung für verschiedene Managementoptionen unter sich ändernden Klimabedingungen.

\section{An wen richtet sich die App?}

1.1. Profil 'Landwirt:innen'

Landwirt:innen, die Ackerbau betreiben und Klimaschutz als wichtig erachten oder direkt in inrer Arbeit bereits berücksichtigen und darüber hinaus technologieaffin sind; sowohl Einzelpersonen als auch Verbände
III Profil 'Politik/Verwaltung'

Ministerien, nachgeordnete Behörden, Kammern und Institutionen mit landwirtschaftlichen Bildungs-, Beratungs-, Förder- und Verwaltungsaufgaben; von Bundes- bis Regionalebene 


\section{Bodenkohlenstoff-App}

\section{Was macht die App besonders?}

- Abschätzung von Potentialen zur Bodenkohlenstoffspeicherung in Kombination mit Klimawandelinformationen für Deutschland

- Informationen für sowohl Mitigationspotential unter verschiedenen Klimaszenarien als auch Information zu den zu erwartenden Klimaänderungen für Klimaanpassungsoptionen

- Flächendeckend, relativ hohe Auflösung als Modeloutput zu Bodenkohlenstoff und Klima; nach Eingabe des Standortes erhalten Nutzer:innen Informationen zu Bodenkohlenstoff und Klima in inrer Region

- Verteilte Service-Architektur: technische Konzepte zu Datenprozessierung, -speicherung und -transfer ermöglichen hohes Maß an Automatisierung, Flexibilität und Übertragbarkeit 


\section{Bodenkohlenstoff-App}

\section{Umsetzung: Aufbau und Struktur}

\section{Technischer Aufbau}

Bodenkohlenstoff: Berechnung verschiedener Landmanagement-Szenarien mit dem

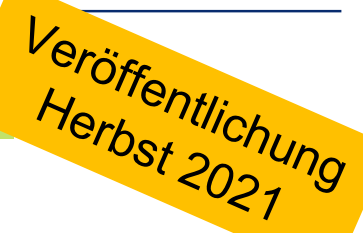
Landoberflächenmodell CLM angetrieben mit verschiedenen Klimaprojektionen (EUROCORDEX-Teilensemble)

Klimainformationen: Analyse von Klimaparametern aus EURO-CORDEX Ensemble Automatisierter Workflow: Rasterdatenbank, Webservice, plattformübergreifendes Frontend voneinander unabhängige Module innerhalb der Architektur

\section{Struktur}

Zwei Informationsebenen:

" "Applikationsebene" $\rightarrow$ grafische Darstellung

- "Wissensebene" $\rightarrow$ textbasierte Darstellung

-

Bodenkohlenstoff App

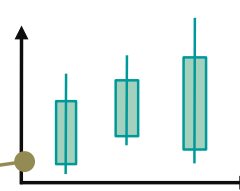

Was bedeutet das? 


\section{Netto-Null-2050 Web-Atlas}

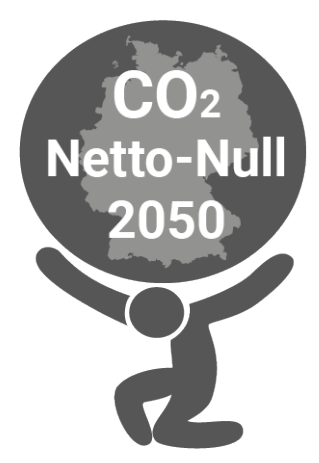




\section{Nationaler Netto-Null-2050 Web-Atlas}

\section{Ziel und Nutzer:innengruppen}

\section{Spezifisches Ziel}

- Beantwortung der Frage: Wo in Deutschland können/müssen wir wie und wie viel $\mathrm{CO}_{2}$ vermeiden/entnehmen, um im Jahr 2050 bei Netto-Null zu sein?

\section{An wen richtet sich der Web-Atlas?}

\section{ZIELGRUPPE}

- Interessierte Fachöffentlichkeit

- Umweltpolitik auf Bundes-, Länder- und Regionalebene

- kommunale Sachverständige/ Fachexpertinnen

\section{INTENTION}

- Informieren und engagieren der Nutzer:innen

- Bereitstellung verschiedener Informationsebenen: Einstiegs-, Strategie-, Experten-Ebene 


\section{Nationaler Netto-Null-2050 Web-Atlas}

\section{Was macht den Web-Atlas besonders?}

\section{Nutzen für die Helmholtz-Klima-Initiative:}

- Web-basiertes WissenschaftskommunikationsProdukt für Netto-Null-2050, Cluster I

\section{Nutzen für Zielgruppen:}

- Projektübergreifender homogenisierter Web-Atlas

- Karten-basierte Inhalte und Story-basierte Inhalte

- Informationsebenen-Konzept

- Einstiegs-, Strategie-, Experten-Ebene

- weitgehend barrierefrei

\section{Einstiegs-Level}

- Hauptthemen in drei Bullet-Points.

- Überblick durch Leitfragen:

- Was zeigt die Karte?

- Warum ist dieses Thema wichtig für Netto-Null 2050?

- Für wen sind die Ergebnisse relevant?

- Storytelling

- Erzählerische Struktur für Graphik-gebundene Inhalte.

Strategie-Entwicklung Level

- Möglichkeiten und Herausforderungen

- National-Roadmap Synthese-Information ]

Experten Level

- detaillierte Informationen in Text und Grafiken

- Links zu Fachbeiträgen

- Links zu weiteren Informationen und Plattformen 


\section{Nationaler Netto-Null-2050 Web-Atlas}

\section{Technischer Aufbau}

Backend: Django-CMS, Geo-Server, SQL-Datenbank

- Uploader-Bereich für Projektpartner:innen:

- Formular-basierte Eingabe der Inhalte

- partiell: CSV-import einer Tabelle

Frontend: Modulare Karten-Graphik-Text Struktur

\section{Struktur}

\section{Modularer Aufbau in Kapiteln:}

- Netto-Null Methoden

- Netto-Null Szenarien

- UNFCCC-Roadmaps-Synthese

- Netto-Null Partner
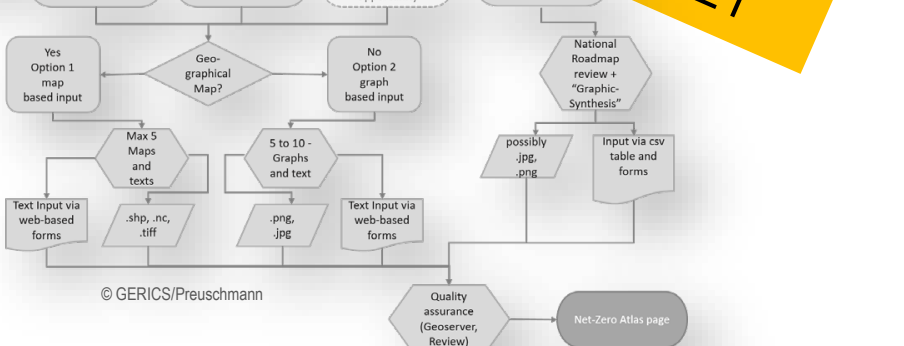


\section{Herausforderungen für die Kommunikation}

\section{Bodenkohlenstoff-App und Netto-Null Web-Atlas}

- viele Daten, viele Kombinationsmöglichkeiten:

- Postprocessing: Raum, Zeit, Variablen, Landmanagementoptionen, Klimaszenarien

- Darstellungsebene: Karten, Graphiken, Illustrationen, Tabellen, usw.

- Vereinfachung des Komplexen:

- Nutzer:innen erwarten einfache und gut verständliche Anwendung, die dennoch wissenschaftlich korrekt ist

- unterschiedliche Zielgruppen:

- wie leiten wir die verschiedenen Nutzer:innen hindurch? 


\section{HELMHOLTZ}

\section{: CLIMATE INITIATIVE}

\section{Vielen Dank für Ihre Aufmerksamkeit}

Copyrighthinweise:

Folie 2: Deutschlandkarte mit eingezeichneten Standorten beteiligter Zentren -

https://www.helmholtz-klima.de: @ Hermann von Helmholtz-Gemeinschaft Deutscher

Forschungszentren e.V.

Folie 3: @ GERICS/Steuri

Folie 4,5,6,7: App-wireframe @ 2008-2021 Balsamiq Studios, LLC - free version bearbeitet Blome, App-View:@ Microsoft PowerPoint bearbeitet Blome\&El Zohbi Folie 5: Icons: @ Microsoft PowerPoint

Folie 4,8,9,10,11: Atlas-Icon und Atlas-View: @ GERICS/Preuschmann

Folie 9: Icons: ๑ Pixabay, bearbeitet Preuschmann

Folie 10,11: Graphiken @ GERICS/Preuschmann 